\title{
Breathing Difficulties - A Problem Among Adolescent Athletes
}

\author{
Kamila Malewska-Kaczmarek ${ }^{1}$, Katarzyna Gmachowska ${ }^{1}$, Katarzyna Makos ${ }^{1}$, Daniela \\ Podlecka $^{1}$, and Joanna Jerzynska ${ }^{1}$ \\ ${ }^{1}$ Medical University of Lodz
}

June 10, 2020

\begin{abstract}
Adolescent athletes are a particular group requiring special medical attention. Recent literature outlines the growing problem of physical inactivity among young people, exploring various dimensions that may explain lack of physical activity, and potentially relevant interventions and strategies. There is growing evidence that physical inactivity predisposes to the development of asthma. Exercise is one of the most common factors triggering cough, dyspnea, and breathing difficulties in otherwise healthy children. This condition is called exercise-induced bronchoconstriction (EIB). Symptoms of EIB include cough, wheezes, chest tightness, and dyspnea during or after physical education lessons. The number of adolescents suffering from EIB is underestimated and many athletes remain undiagnosed and untreated. In this article we argue that complexity of diagnosing EIB is greater for adolescent athletes than it is for adults. The purpose of this review is to draw the attention of the medical community to the problem of EIB and asthma symptoms among adolescent athletes. We performed a meta-analysis of these two topics, consulting PubMed and ClinicalKey for the terms: "adolescent athletes", "exercise-induced bronchoconstriction" and "asthma in adolescent athletes". After analysis of the relevant scientific data, the most important facts and issues related to the study group were listed.
\end{abstract}

\section{Introduction}

Exercise is one of the most common factors triggering cough, dyspnea, or breathing difficulties in otherwise healthy children. This condition is called exercise-induced bronchoconstriction (EIB), a frequent phenomenon among early adolescent athletes. Symptoms of EIB include cough, wheezes, chest tightness, and dyspnea during or after physical education lessons. The number of adolescents suffering from EIB is underestimated and many athletes remain undiagnosed and untreated. In this article we argue that complexity of diagnosing EIB is greater for adolescent athletes than it is for adults, due to a reduced capacity of adolescents to feel and understand symptoms. Furthermore, adolescents experience greater difficulty reporting any such symptoms of EIB to medical authorities. Chronic exposure to bronchospasm factors may be associated with the development of asthma. On the other hand, the beneficial effect of physical effort, especially for children whose symptoms are well-controlled, permits young people to perform physical exercises, even at the elite level ${ }^{1}$.

The purpose of this review is to draw the attention of the medical community to the problem of EIB and asthma symptoms among adolescent athletes. In the available sources, most data pertains to professional adult athletes, so after analyzing the scientific data of the collected material, the most important issues related to adolescent athletes were listed.

\section{History of EIB}

In the second century CE Greek physician Aretaeus the Cappadocian wrote: "If from running, and exercise, and labor of any kind a difficulty of breathing follows it is termed asthma". This may be the earliest known written report of $\mathrm{EIB}^{2}$. More recent information regarding EIB was published in 1860, in a book entitled 
"On Asthma: its pathology and treatment" published by Salter ${ }^{3}$. Salter described various types of asthma with predisposing factors, dividing the disease into two types: "spasmodic" (i.e., idiopathic, "uncomplicated") and "organic" ("complicated"). According to the author, "as exercise level increases, a rapid flow of fresh and cold air through the bronchi triggers organic asthma symptoms".

The quantitative description of bronchospasm during exercise was presented in the 1960s. In certain patients with asthma, researchers observed a decrease in forced expiratory volume in 1 second (FEV1) during and after exercise, describing this phenomenon asexercise-induced asthma (EIA) ${ }^{4}$. In later years the term exerciseinduced bronchospasm was used and finally become exercise-induced bronchoconstriction ${ }^{5}$.

\section{Definition of EIB}

Exercise-induced bronchoconstriction is defined as acute narrowing of the respiratory tract that occurs as a consequence to physical effort. This condition occurs both transiently and reversibly, which means that in a few minutes after physical effort, the condition of the airways returns to the state before exercise. EIB can occur both during and after exercise, and can be manifested by coughing, chest tightness, shortness of breath and wheezing. The greatest reduction of airflow usually occurs approximately 10 to 15 minutes after the start of exercise and may last up to 30 to 60 minutes before resolving. A relative refractory period is also observed, and the repetition of exercise within 60 minutes of the original symptom usually causes recurrence of bronchoconstriction ${ }^{6}$.

Furthermore, this condition can be observed both among healthy patients, especially those performing strenuous physical activity, and those suffering from asthma ${ }^{7}$. For this reason, some researchers suggest that EIA and EIB are synonyms ${ }^{8}$. Others have stated that EIA occurs only in asthma-diagnosed patients, while EIB appears exclusively in those who do not present symptoms of asthma ${ }^{9,10}$.

The question has been raised by some researchers, whether the number of patients suffering from EIB is underestimated and whether many such athletes remain undiagnosed or untreated ${ }^{11}$. Our findings suggest that reduced awareness of symptoms of early adolescents significantly hinders diagnosis of EIB. Dickinson $\mathrm{J}$ et al. state that only $59 \%$ of athletes diagnosed with EIB reported at least one symptom. Other reasons why athletes do not report their symptoms may include concern over losing status in their team or showing "signs of weakness" ${ }^{2}$. Furthermore, athletes may not recognize symptoms of EIB and misinterpret breathing difficulties as a normal part of their physical training. It is also worth noting the variety of EIB symptoms, as in some athletes normal results of respiratory tests are connected with signs of EIB. This finding is related to study by Rundell et al., who suggested that symptoms reported by athletes are not related to the presence of bronchospasm. The proper diagnosis should be based on accurate patient history, spirometry and an exercise challenge test. Many physicians treat EIB only on the basis on symptoms, without performing a standardized exercise test ${ }^{13}$.

\section{Pathogenesis of EIB}

There are two proposed theories - osmotic and thermal - explaining the occurrence of EIB. The osmotic theory assumes that hyperventilation during physical effort leads to loss of water from the surface of the respiratory epithelium, causing disorders in cell ion homeostasis and an increase in mucus osmolarity. Respiratory tract wall contains mast cells and eosinophils, that when stimulated release inflammatory mediators, leading to a form of bronchospasm. A higher concentration of inflammatory cells in the sputum of patients suffering from EIB supports this theory.

The thermal theory, assumes that heat loss of the airways with their subsequent reheating by incoming air after exercise, may lead to an increase in mucus secretion, swelling and inflammation of tissues, which ultimately causes narrowing of the airways. Additionally, cold air stimulates parasympathetic nerve stimulation, which through vagal tone leads to bronchoconstriction with simultaneous constriction of the venous vessels in an attempt to limit heat loss. At the end of physical effort, reflex dilatation of vessels occurs. All these factors ultimately lead to narrowing of the airways, increasing in their air-resistance ${ }^{14}$.

Among competitive athletes practicing winter sports, it is suspected that EIB is caused by chronic exposure 
to cold air during vigorous breathing, which results in damage to the respiratory epithelium and its repair. In summer sports it is suggested that chronic exposure to environmental allergens may be related to elevated circulating levels of $\operatorname{IgE}^{15}$.

EIB is often diagnosed among swimmers and is linked to substances added to pool water. During swimming, athletes inhale chlorine-rich water molecules above the surface of the swimming pool, which irritate the respiratory tract. Repeated exposure to these substances can lead to the inflammation, epithelial damage and development of EIB and asthma.

Other causes of respiratory tract irritation in athletes may be due toenvironmental factors, such as airborne allergens (e.g. pollen). It has been observed that people training in cold air, e.g. hockey, figure skaters and speed skaters, may be more exposed to higher concentrations of nitrogen dioxide $\left(\mathrm{NO}_{2}\right)$ and particles left over by ice leveling machines. Athletes training on the streets, e.g. runners or cyclists, are more likely to inhale polluted street air.

Furthermore, among children who regularly play team games in the open air, there is a greater risk of developing symptoms of asthma, especially in an ozone-rich environment. McConnell et al. observed a greater risk of developing asthma in athletes training more actively and spending more time outside. Hyperventilation and breathing by mouth are conducive to ozone, which progressively reaches more distant parts of the respiratory tract. According to one study, the development of asthma may be associated with neglecting EIB symptoms, which over time may lead to airway remodeling and development of asthma ${ }^{16}$.

\section{Prevalence of EIB in Young Athletes}

In the general population, the EIB frequency is estimated at 5-20\%, in children at 3-35\%. The prevalence among adult elite athletes is higher, especially those at the Olympic-level (30-70\%). These discrepancies may be due to the type of practiced sport, exposure to air pollution and allergens and maximum exercise intensity achieved, which may vary depending on the sport season ${ }^{17}$.

Prevalence of EIB among young competitors is underestimated, but a few studies report that the incidence of EIB in this group varies from $12 \%$ to $38 \%$. Jonckheere AC reports the prevalence of EIB in $33.3 \%$ swimmers, $27.3 \%$ basketball and $12.8 \%$ football players ${ }^{18}$. Among young high school and college athletes in Mexico City, the EIB frequency was set at $7.2 \%$, no differences between male and female sex were observed. Ventura et al. indicated a frequency of self-reported EIB symptoms in young soccer players at $5.1 \%{ }^{19}$. On the contrary, Bougault et al. reported $16 \%$ of EIB in young soccer players. Among the undiagnosed young athletes suspected of EIB, the incidence was $29 \%^{20}$.

A high, $42.9 \%$ EIB prevalence was demonstrated in young weightlifters. Authors claim that EIB is usually associated with sports provoking elevated minute ventilation, but in this case repetitive Valsalva maneuvers may be a risk factor in EIB diagnosis.

\section{Diagnosis of EIB}

Diagnosis of EIB among young athletes does not presently differ from adult athletes. In the physical examination, especially at rest, patients suffering from EIB do not exhibit abnormalities. Diagnosis can be made when respiratory symptoms occur during exercise and are linked to confirmation with an exercise challenge test, performed by a standardized protocol.

Several tests can be used to assess the occurrence of bronchospasm in EIB-positive athletes. One such test is spirometry, which usually does not rule-out the occurrence of EIB and can give false negative results.

Regular exercise is a factor that improves functions of the respiratory tract. Athletes are characterized by higher cardiovascular and respiratory system parameters, meaning their baseline spirometry parameters can be higher than in general population ${ }^{21}$.

Provocation tests provide a basis for diagnosing bronchospasm during exercise and are divided into two groups: direct andindirect. The first group contains a metacholine challenge, which is direct bronchoprovo- 
cation test, designed to stimulate a response of the respiratory tract to the administration of methacholine. A fall in FEV1 [?] 20\% from the baseline value is considered positive.

Indirect tests consist of an exercise challenge via treadmill (in accordance with the recommendations of the American Thoracic Society, ATS), a free running exercise challenge, eucapnic voluntary hyperventilation $(\mathrm{EVH})$, hypertonic saline challenge, and the mannitol test.

The most recommended by scientific societies is the exercise challenge on treadmill or a cycle ergometer, which consists of six to eight minutes of physical effort, with a heart rate of $80 \%$ to $90 \%$. Respiratory response is measured by performing repetitive spirometry, usually after 5, 10, 15 and 30 minutes of exercise. The test is considered positive with decline in FEV1 [?] $10 \%$ from the baseline value, according to the recommendations of the American Thoracic Society ${ }^{22}$.

The free running exercise is based on repetitive peak expiratory flow (PEF) measurements after physical exercise lasting approximately 6 minutes, but mainly depends on the subject's motivation to complete the test with maximal effort ${ }^{23}$.

Eucapnic Voluntary Hyperventilation $(\mathrm{EVH})$ is a test specially developed for EIB diagnosis that indirectly causes bronchospasm. During the test, a patient is required to breathe for six minutes at $85 \%$ maximum voluntary ventilation rate with a controlled concentration of carbon dioxide. Inhaled air should contain $21 \%$ oxygen, $5 \%$ carbon dioxide and $74 \%$ nitrogen. After three, five, 10 and 15 minutes, maximal voluntary flow-volume loops should be assessed. As in the exercise challenge on treadmill, EVH is considered positive when FEV1 [?] 10\% decreases from baseline value. Good standardization and sensitivity are the reasons for which EVB is the preferred diagnostic test used by many researchers. EVH is limited by its requirement for special equipment and more demanding procedures, including proper training of testing personnel.

The hypertonic saline challenge and mannitol tests are indirect tests that involve the inhalation of hypertonic saline and mannitol, respectively. The tests are considered positive when there is a fall in the baseline value in FEV1 [?] $15 \%^{24,25}$.

\section{Differential Diagnosis}

According to The American College of Chest Physicians (CHEST) guidelines and Expert Panel Report, the differential diagnosis of cough occurring in athletes over 12 years of age are EIB, asthma, respiratory tract infections and upper airway cough syndrome due to rhinosinusitis. Environmental factors that may significantly affect lung function should also be taken into consideration ${ }^{26}$.

In a differential diagnosis of a young athlete presenting with breathing difficulties, laryngeal wheeze during physical effort, dyspnea and cough, exercise-induced vocal cord dysfunction (VCD) should also be taken into consideration. Exercise-induced VCD affects up to 5-27\% of patients earlier diagnosed with EIB. In such cases during physical effort, paradoxical motion of the vocal cords occurs, especially during inspiration ${ }^{27}$. Particularly, patients with difficult-to-treat asthma and athletes whose symptoms do not improve after treatment should be considered in the differentiation from exercise-induced VCD. Exercise-induced laryngomalacia may be another presenting condition. In both cases FEV1 does not decrease after exercise or after the administration of 32 -mimetics and / or inhaled GKS.

Dysfunctional breathing (activation of additional inspiratory muscles, with shallow breathing, and lifting of the chest wall), acquired and habitual patterns of breathing, should also be considered in differentiating EIB among young athletes. Dysfunctional breathing often occurs in people with asthma. It is characterized by the absence of any functional airway disorder, exercise-induced hyperventilation, and often co-occurs with acute anxiety. As a result of hyperventilation, respiratory alkalosis occurs, causing dyspnea, light-headedness, chest tightness and paraesthesias.

When investigating dyspnea, it is widely emphasized to take psychological factors into consideration, especially when working with young patients. This is because dyspnea is a subjective measurement and the symptoms that occur during exercise can be interpreted differently. 
Rare pathologies causing exercise-induced symptoms, especially in adolescent athletes, include hypertrophic cardiomyopathy (HCM), which is the main cause of sudden cardiac death in this group. Other rare pathologies that simulate EIB symptoms include: exercise-induced arrhythmia, cardiovascular dysfunctions, pulmonary embolism, anemia, interstitial lung disease, swimming induced pulmonary edema, exercise-induced anaphylaxis, and myopathies.

\section{Young Athletes in the Light of New Threats}

At the end of 2019 few expected a new coronavirus - SARS-CoV-2 - whose outbreak appeared in Wuhan, China, would significantly affect the world population. After the World Health Organisation (WHO) announced a pandemic in March 2020, there was concern about patients who suffer from chronic diseases, including those with respiratory symptoms. Due to the on-going phase of the pandemic at the time of writing, we are likely to see many new research developments. In this section, we discuss a few early results.

Presently there is no evidence that asthma in children is a risk factor for infection with coronavirus disease 2019 (COVID-19). However, symptoms of COVID-19 can mimic asthma exacerbation, including dry cough and shortness of breath. Fever, which commonly occurs in COVID-19, could be a differentiating factor from asthma exacerbation. Furthermore, epidemiologic information such as travel history and possible contact with infected persons should be considered. From the limited data from the United States, children with symptoms of asthma should continue therapy. This approach is supported by the statement of the Global Initiative for Asthma (GINA), the Centers for Disease Control and Prevention and the North American Consensus Guideline on Allergy Care. Additional recommendations include frequent handwashing, apply the principle of social distancing, and avoiding airborne allergens.

Special attention should be paid to athletes whose sports plans were cancelled or postponed by this pandemic, including those with EIB and asthma. There is currently no data available on prevalence, nature and behavior of COVID-19 in athletes.

Consequences of isolation favor increased sedentary behaviors, lack of communication among athletes and coaches, and an insufficient amount of exercise. Negative lifestyle changes can lead to inappropriate nutrition and an increase in body fat, which affects both physical and mental health. There is a global consensus that the beneficial effect of regular physical effort at moderate volume and intensity is associated with decreased risk of respiratory infections. A sedentary lifestyle poses a greater threat to athletes who depend on daily exercise. The restrictions put in place by governments to prevent spread of COVID-19, limit athletes' ability to train. At the time of writing COVID-19 symptoms reported in pediatric patients are relatively mild with recovery lasting 5-7 days. Nevertheless, a prolonged rest period for such patients is recommended.

Jukic et al. offer strategies and solutions for athletes during the COVID-19 pandemic. Firstly, they recommend encouraging athletes to re-adapt their approach to isolation as an opportunity for "personal development." Secondly, by using the proper technology, athletes should be supported and be in constant contact with experts, such as coaches, nutritionists, physicians and psychologists. Adequate equipment should be provided at the athletes' living conditions, such as cardio equipment (e.g. treadmill, bicycle), resistance training equipment (e.g. dumbbells, elastic or medicine bands), and other techniques, which the athlete can use to support exercise (e.g. mats, foam rollers). Alternative sports skills training should be considered (e.g. kinesthetic ball training in small space). The physician's role is to educate athletes about nutrition, supplementation, and hydration, and also to encourage "preventive behaviors" (e.g. washing hands, social distancing). Every athlete should use forms of self-assessment and establish daily monitoring by the use of modern technology ${ }^{28}$.

To summarize, athletes should comply with limitations and apply strategies that will allow them to maintain an appropriate level of physical exertion. Those, who have asthma symptoms should remain on their current medication and introduce detailed control over the treatment. More research will be required to help improve these guidelines in the coming months ${ }^{29,30}$.

\section{A Growing Problem with Allergies}


The majority of research related to sports and allergy focus on adult professional athletes, with few studies based on young athletes. Ventura et al. analyzed population of young soccer players and reported high prevalence of allergic diseases in this group. However, in the comparison with control students, results were not significantly different. This suggests that intensive training by soccer players is not related to a greater risk of allergic disease.

Bougault et al. reported that $49 \%$ of studied soccer players were allergic to at least 1 allergen, $33 \%$ reported allergies and $16 \%$ had confirmed EIB. Rates of allergy treatment was low, mainly because $44 \%$ of athletes believed that antiallergic drugs harmed their physical performance and could disqualify them from training, due to the possible presence of these drugs on World Anti-Doping Agency (WADA) prohibited list. The study emphasizes the necessary not only to diagnose players with respiratory symptoms, but also to screen asymptomatic athletes.

\section{Quality of Life in Young Athletes}

Johansson et al. reported that compared to healthy adolescents, adolescents with EIB have more absences from school and respiratory symptoms during exercise, factors that may limit their motivation to improve physical activity ${ }^{31}$.

Based on the Health-Related Quality of Life Survey (HRQoL), young girls who suffer from EIB scored lower in both respiratory function tests and HRQoL when compared to a population of EIB-negative girls. Such a relationship was not observed in boys. Moreover, girls with confirmed EIB had more sleep disturbances and the results in anxiety scores were significantly higher, compared to the healthy population ${ }^{32}$. Hallstrand et al. report that HRQoL results were significantly lower among adolescent athletes with previously confirmed EIB, mild asthma and co-existing allergic rhinitis. Furthermore, the symptoms of dyspnea that appeared during exercise had a significant impact on the HRQoL result. Researchers suggest that proper treatment can improve the results of the survey and the quality of life of young people ${ }^{33}$.

Among the surveyed children and adolescents at the EIB Landmark Survey, US researchers found a higher prevalence of children avoiding exercises $(31.8 \%)$, due to exercise-related symptoms ${ }^{34}$.

However, when children with asthma symptoms took part in physical exercise, their quality of life improved. Vahlkvist et al. reported less physical fitness in newly diagnosed asthmatic adolescents compared to control subjects. After one year of treatment, control of the disease and proper treatment both increased physical activity $^{35}$.

\section{Non-Pharmacological Help for Young Athletes}

Reducing symptoms of bronchospasm is not limited to the use pharmacological methods. One of the nonpharmacological methods applicable to young athletes is proper warm-up to induce a refractory period in the respiratory tract. Methods that are designed to moisturize and pre-warm air during exercise can reduce the risk of bronchoconstriction. It is especially recommended to use face masks in cold air conditions during training and sports competitions. According to ATS guidelines, proper warm-up before planned exercise along with 10-15 minutes of moderately vigorous exercise (high-intensity interval or variable intensity warmup exercise) can help to reduce this risk. In addition, recommendations include limitation of exposure to allergens and air pollutants.

Studies showed smaller decrease in FEV1 during physical exercise, which is preceded by high-intensity interval or variable intensity warm-up, compared to no warm-up ${ }^{36}$.

\section{Pharmacological Methods of EIB Treatment}

The ATS guidelines for the general population with EIB showed that EIB can be present both in patients with and without confirmed asthma. They recommend taking a short-acting $\beta 2$-agonist (SABA) inhalation 520 minutes prior to a planned exercise. When SABA dosing is more frequent but the patient still suffers from EIB symptoms, daily monotherapy with inhaled corticosteroids (ICS) is recommended, which are considered the most effective anti-inflammatory agents in EIB treatment. A long-acting $\beta 2$-agonist (LABA) may be 
used if necessary. Among the drugs with protective effects against EIB, formoterol (LABA) in dry powder inhalation can prevent EIB within a few minutes after administration for a duration of at least 4 hours ${ }^{37}$. Among athletes who rarely experience EIB episodes, it is sufficient to administer SABA before exercise.

Daily use of a leukotriene receptor antagonist (LTRA) is also recommended. It should be taken about two hours before planned exercise, however its effects are smaller than in the case of SABA or ICS. As a secondline therapy, it is possible to use inhaled anticholinergic agents or a mast cell stabilizing agent before exercise. Among patients suffering from EIB and allergies, who use SABA only before exercise or in patients with daily and more frequent use of SABA - addition an antihistamine is recommended.

Ducharme F. emphasizes that for adults with a mild or moderate asthma it has been demonstrated that equivalent to $400 \mathrm{~g} /$ day beclomethasone is more effective than leukotriene receptor antagonists. The authors suggest that among treated children there is insufficient evidence to detect the efficacy of leukotriene antagonists, but others argue that anti-leukotriene drugs should remain a treatment option for the athletes with EIB when SABA and ICS therapy is insufficient ${ }^{38}$.

\section{The World Doping Agency}

The World Doping Agency (WADA), an agency established in 1999 by the International Olympic Committee (IOC), fights doping among athletes and protects the integrity of sport against the use of illicit substances. Every year WADA publishes a list of prohibited drugs, which includes $\beta 2$-agonists and ICS, treatments for athletes suffering from EIB and asthma. These substances are considered to be performance-enhancing drugs.

Although these drugs are not allowed to be used in systemic administration by athletes, under certain conditions, inhalation use is permitted for athletes who suffer from EIB and asthma. The IOC regulations require diagnosis of AHR or reversibility to bronchodilators, to allow the use of such drugs during competition. Pharmaceuticals from this group include (inhaled): formoterol, salbutamol, terbutaline and salmeterol. Furthermore, WADA or the IOC Medical Commission must always be informed of which athletes are using ICS treatment.

\section{Summary}

Our review has revealed that EIB is under-diagnosed and under treated in young athletes. An objective diagnosis of EIB should be based on symptoms in a combination with a proper exercise challenge test, as athletes who refrain from reporting symptoms experience a decline in lung function performance after exercise.

Nonetheless, it is important to avoid over-diagnosing EIB, which is usually followed by over- or undertreatment. Limited access to relevant, provocation tests can contribute to a lack of awareness and misdiagnosis of EIB, as asthma and other diseases produce similar symptoms.

Appropriate treatment for the diagnosis of EIB in young athletes should be associated with regular control visits.

\section{Acknowledgments:}

The study was supported grant 503/1-047-01/503-21-001-18 from the Medical University of Lodz, Poland.

\section{Conflict of interest:}

All authors state no conflict of interests

\section{References:}

1. Wuestenfeld JC, Wolfarth B. Special considerations for adolescent athletic and asthmatic patients. Open Access J Sport Med 2013;4:1-7.

2. Adams F. 1856. The Extant Works of Aretaeus the Cappadocian. Syndenham Society. 510 p.

3. Salter HH. 1860. On asthma: Its Pathology and Treatment. London: John Churchill. 464 p. 
4. Jones RS, Wharton MJ, Buston MH. The Place of Physical Exercise and Bronchodilator Drugs in the Assessment of the Asthmatic Child. Arch Dis Child 1963;38(202):539-545.

5. Aggarwal B, Mulgirigama A, Berend N. Exercise-induced bronchoconstriction: prevalence, pathophysiology, patient impact, diagnosis and management. NPJ Prim Care Respir Med 2018;28(1):31.

6. Tilles SA. Exercise-induced respiratory symptoms: an epidemic among adolescents. Ann Allergy Asthma Immunol 2010;104(5):361-367.

7. Becerril-Ángeles M, Vargas MH, Hernández-Pérez L, Rivera-Istepan NJ, Pérez-Hidalgo RI, OrtegaGonzález AG, Rubio-Domínguez S, Rodríguez-Gutiérrez MC, Gaxiola-Cortés R, Dosal-Ulloa R, et al. Prevalence and characteristics of exercise-induced bronchoconstriction in high school and college Athletes at 2,240 m altitude. Rev Investig Clin 2017;69(1):20-27.

8. Gotshall RW. Exercise-Induced Bronchoconstriction. Drugs 2002;62(12):1725-1739.

9. Hildebrand K. Exercise-induced bronchoconstriction. Pneumonol Alergol Pol 2011;79(1):39-47.

10. Weiler JM, Bonini S, Coifman R, Craig T, Delgado L, Capão-Filipe M, Passali D, Randolph Ch, Storms W, Ad Hoc Committee of Sports Medicine Committee of American Academy of Allergy, Asthma \& Immunology. American Academy of Allergy, Asthma \& Immunology Work Group Report: Exerciseinduced asthma. J Allergy Clin Immunol 2007;119(6):1349-1358.

11. Bougault V, Drouard F, Legall F, Dupont G, Wallaert B. Allergies and Exercise-Induced Bronchoconstriction in a Youth Academy and Reserve Professional Soccer Team. Clin J Sport Med 2017;27(5):450456.

12. Dickinson J, McConnell A, Whyte G. Diagnosis of exercise-induced bronchoconstriction: Eucapnic voluntary hyperpnoea challenges identify previously undiagnosed elite athletes with exercise-induced bronchoconstriction. Br J Sports Med 2011;45(14):1126-1131.

13. Rundell KW, Im J, Mayers LB, Wilber RL, Szmedra L, Schmitz HR. Self-reported symptoms and exercise-induced asthma in the elite athlete. Med Sci Sports Exerc 2001;33(2):208-213.

14. Carlsen KH. The breathless adolescent asthmatic athlete. Eur Respir J 2011;38(3):713-720.

15. Anderson SD, Kippelen P. Airway injury as a mechanism for exercise-induced bronchoconstriction in elite athletes. J Allergy Clin Immunol 2008;122(2):225-235.

16. McConnell R, Berhane K, Gilliland F, London SJ, Islam T, Gauderman WJ, Avol E, Margolis HG, Peters JM. Asthma in exercising children exposed to ozone: a cohort study. Lancet 2002;359(9309):896.

17. Durmic T, Lazovic B, Djelic M, Suzic Lazic J, Zikic D, Zugic V, Dekleva M, Mazic S. Sport-specific influences on respiratory patterns in elite athletes. J Bras Pneumol 2015;41(6):516-522.

18. Jonckheere AC, Seys S, Dilissen E, Schelpe AS, Van der Eycken S, Corthout S, Verhalle T, Goossens J, Vanbelle V, Aertgeerts S, et al. Early-onset airway damage in early-career elite athletes: A risk factor for exercise-induced bronchoconstriction. J Allergy Clin Immunol 2019;144(5):1423-1425.e9.

19. Ventura MT, Cannone A, Sinesi D, Buquicchio R, Carbonara M, Di Leo E, Bonini M, Dagnello M, Bonini S. Sensitization, asthma and allergic disease in young soccer players. Allergy 2009;64(4):556-559.

20. Rupp NT, Guill MF, Brudno DS. Unrecognized Exercise-Induced Bronchospasm in Adolescent Athletes. Am J Dis Child 1992;146(8):941-944.

21. Azmeh R, Greydanus DE, Agana MG, Dickson CA, Patel DR, Ischander MM, Lloyd RD Jr. Update in pediatric asthma: Selected issues. Dis Mon 2020;66(4):100886.

22. Parsons JP, Hallstrand TS, Mastronarde JG, Kaminsky DA, Rundell KW, Hull JH, Storms WW, Weiler JM, Cheek FM, Wilson KC, et al. An official American thoracic society clinical practice guideline: Exercise-induced bronchoconstriction. Am J Respir Crit Care Med 2013;187(9):1016-1027.

23. Powell CV, White RD, Primhak RA. Longitudinal study of free running exercise challenge: Reproducibility. Arch Dis Child 1996;74(2):108-114.

24. Mai XM, Nilsson L, Kjellman NIM, Björkstén B. Hypertonic saline challenge tests in the diagnosis of bronchial hyperresponsiveness and asthma in children. Pediatr Allergy Immunol 2002;13(5):361-367.

25. Porsbjerg C, Sverrild A, Backer V. The usefulness of the mannitol challenge test for asthma. Expert Rev Respir Med 2013;7(6):655-663.

26. Boulet LP, Turmel J, Irwin RS, CHEST Expert Cough Panel. Cough in the Athlete: CHEST Guideline and Expert Panel Report. Chest 2017;151(2):441-454. 
27. Mazurek H, Sawiec P. Dolegliwości ze strony układu oddechowego zwiazane z wysiłkiem. Med Prakt Pediatr 2019;01:61-71.

28. Jukic I, Calleja-González J, Cos F, Cuzzolin F, Olmo J, Terrados N, Njaradi N, Sassi R, Requena B, Milanovic L, et al. Strategies and Solutions for Team Sports Athletes in Isolation due to COVID-19. Sports $2020 ; 24 ; 8(4)$.

29. Abrams EM, Szefler SJ. Managing Asthma during COVID-19 An Example for Other Chronic Conditions in Children and Adolescents. J Pediatr 2020; doi: 10.1016/j.jpeds.2020.04.049

30. Hull JH, Loosemore M, Schwellnus M. Respiratory health in athletes: facing the COVID-19 challenge. Lancet Respir Med 2020; doi: 10.1016/S2213-2600(20)30175-2.

31. Johansson H, Norlander K, Hedenström H, Janson C, Nordang L, Nordvall L, Emtner M. Exerciseinduced dyspnea is a problem among the general adolescent population. Respir Med 2014;108(6):852858.

32. Johansson H, Norlander K, Janson C, Malinovschi A, Nordang L, Emtner M. The relationship between exercise induced bronchial obstruction and health related quality of life in female and male adolescents from a general population. BMC Pulm Med 2016;16(1):63.

33. Hallstrand TS, Curtis JR, Aitken ML, Sullivan SD. Quality of Life in Adolescents with Mild Asthma. Pediatr Pulmonol 2003;36(6):536-543.

34. Ostrom NK, Eid NS, Craig TJ, Colice GL, Hayden ML, Parsons JP, Stoloff SW. Exercise-induced bronchospasm in children with asthma in the United States: Results from the Exercise-Induced Bronchospasm Landmark Survey. Allergy Asthma Proc 2011;32(6):425-430.

35. Vahlkvist S, Pedersen S. Fitness, daily activity and body composition in children with newly diagnosed, untreated asthma. Allergy 2009;64(11):1649-1655.

36. Stickland MK, Rowe BH, Spooner CH, Vandermeer B, Dryden DM. Effect of warm-up exercise on exercise-induced bronchoconstriction. Med Sci Sports Exerc 2012;44(3):383-391.

37. Ferrari M, Balestreri F, Baratieri S, Biasin C, Oldani V, Lo Cascio V. Evidence of the rapid protective effect of formoterol dry-powder inhalation against exercise-induced bronchospasm in athletes with asthma. Respiration 2000;67(5):510-513.

38. Ducharme FM. Inhaled glucocorticoids versus leukotriene receptor antagonists as single agent asthma treatment: systematic review of current evidence. BMJ 2003;326(7390):621. 